\title{
Soft Robotics in Medicine
}

\author{
Anthony Donaldson
}

Stephenson School of Biomedical Engineering, University of Oklahoma, Norman OK, 73071

\begin{abstract}
Robots are seeing increasing integration into the medical field, but they are often limited by their size, lack of flexibility, and other intrinsic impediments. However, an area of recent interest, soft robotics, has the potential to overcome these limitations, as soft robots are inherently flexible and compliant. These traits allow them to effectively navigate tight spaces and complex geometry, such as the interior of the human body. Additionally, the lack of distinction between structural members and actuators displayed by soft robots allows for a great deal of customization in their structure and function, with their so-termed soft actuators having a wide variety in composition and mode of activation. As such, it is conceivable that these soft robots, given enough time and research, will be able to solve many issues facing the medical field, such as targeted or sustained drug release, and improve many areas of medicine, through applications such as minimally invasive surgery.
\end{abstract}




\section{Introduction}

From phones to cars and from manufacturing to healthcare, computers and robots are seemingly ubiquitous in today's society. While many areas have taken full advantage of robotic technologies, the medical field has been one of the slowest to integrate robotics into its practice, and understandably so. As people's lives will often hang in the balance, directly involving robots in healthcare requires all parties involved to have a high degree of trust in the technology being used, meaning the technology itself must be vigorously tested and free of virtually any bugs and glitches. From a more ergonomic standpoint, surgical robots must have space to function within operating rooms, which may not always be spacious enough to accommodate oversized machinery. Nonetheless, research into surgical applications of robotics has persevered, and one emerging robotic solution is that of soft robotics. Unlike their traditional, "hard" counterparts, soft robots are, as the name states, inherently soft: composed of highly compliant and elastic materials that allow them to move with considerably more freedom than traditional robots. These properties also have the potential to lend greater viability in surgery, as they may be able to function better in cluttered or constrained areas. In these ways, soft robotics stands to revolutionize the concept of robotic surgery and perhaps medicine as a whole. This paper will discuss soft robotics, its applications to medicine, and recent advances in the field - particularly in actuator materials.

\section{Soft Robotics}

\subsection{Robotics in Medicine}

The use of robots in medical procedures is not an especially novel idea. In fact, researchers have been experimenting with this concept for more than 35 years, with the first surgical application of robotics occurring in 1985 [1]. This initial foray into robotic surgery served as the predecessor to many other robotic surgical devices, including devices that can take brain biopsies or core out the femoral head for hip replacements, although the latter is not FDA-approved as of now [1]. These surgical devices are a couple examples of traditional (or "hard") robots: robotic devices that are composed of a hard, rigid structure [2]. 
While these devices do provide an increase in accuracy, consistency, and reliability, they also tend to come with several drawbacks. In particular, hard robots often have difficulty functioning in unstructured or crowded work areas and require a specifically designed "end effector" to execute their function [2]. Particularly in medical applications, it is highly desirable for a robot to be able to function properly in confined areas (e.g. to navigate around a patient's anatomy). As such, researchers have worked to develop robots that are more robust than traditional hard robots.

\subsection{Advantages and Disadvantages of Soft Robots}

Until recently, researchers in the field of robotic surgery have been limited to working with hard robots exclusively. However, in recent decades, there has been increasing interest in developing a new kind of robot, one that is more robust and flexible with confined environments [2]. This has resulted in the creation of the field of soft robotics.

Soft robots are composed of highly compliant materials and are as a rule much less rigid than their traditional predecessors, hence the name [2]. Additionally, soft robots are capable of a much wider range of motion, allowing them to retain functionality in more confined spaces than hard robots. This ability to navigate obstacles and confined areas is referred to as dexterity. For soft robots, a high degree of dexterity is possible because the techniques and mechanisms employed in soft robots allow them to theoretically obtain infinitely many degrees of freedom (a measure of how much and in what direction the machine can move), whereas hard robots are much more limited in their range of motion by a finite set of movement points (e.g. pneumatic pistons, joints) [2].

However, this limitation in traditional robots' ranges of motion also provides them with some benefits: easier tracking and greater accuracy [2]. While the immense range of motion granted by softer robots provides for much greater dexterity, flexibility, and adaptability, it also makes them very difficult to 
track (see Fig. 1) [2]. Unlike soft robots, hard robots generally have a discrete amount of movement points and ways in which each of those points can move, allowing their endpoints to be easily tracked. With soft robots however, position tracking becomes increasingly difficult with the increased quantity of degrees of freedom, and as a result, the device's accuracy may

(a)

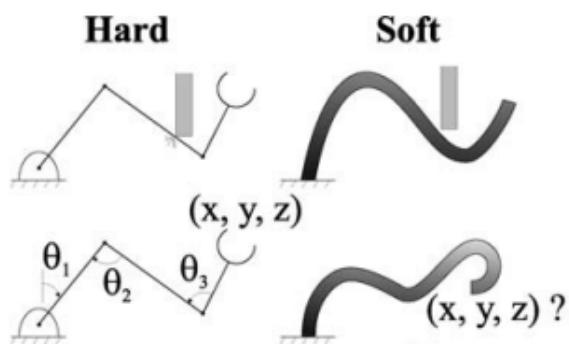

Figure 1: Comparison of hard and soft robotics in terms of (a) dexterity and (b) positional tracking. Borrowed from Trivedi et al. [2].

suffer. Such devices generally rely on various sensors to measure deformation, but even these can leave room for error and output data that can be difficult to integrate into tracking algorithms $[2,3]$.

Furthermore, hard robots tend to outperform their soft counterparts in terms of load bearing, but again, this is a bit of a trade-off. The rigid structures used in hard robots are inherently more conducive to supporting large masses than are the more compliant structures utilized by soft robots. The materials used in hard robots (generally metal and/or stiff plastics) are strong enough to support large loads (such as car parts in automotive manufacturing) and effectively absorb vibrations (such as those resulting from coring the femoral head for hip replacement) without experiencing a decrease in accuracy but are unable to navigate obstructions, at least not without a significant degree of difficulty [1,2]. On the other hand, soft robots are generally composed of rubber and other deformable polymers that allow them to move with greater freedom but limit their load-bearing capabilities [2]. Furthermore, their greater degree of motion allows soft robots to perform multiple different end functions without needing different tools, which allows them to manipulate objects of varying sizes and geometries, whereas hard robots generally can only work with objects within a limited size range [2]. As such, the application of hard or soft robotics to a situation - even in medicine - largely depends on the problem itself. For a situation that requires a high load-bearing capability or high force output, a hard robot may be more desirable, whereas a soft robot may be more appropriate for applications that are less work-heavy and demand more navigability and/or the ability to manipulate objects of variable shapes and sizes.

\subsection{Biological Inspiration}


As with many research areas, soft robotics researchers often look to nature for inspiration. Examples of "soft" manipulators are abundant: elephant trunks contain no bone yet are able to support more than 700 pounds; the Venus flytrap (Dionaea muscipula) is able to close its "mouth" in less than a second despite having no muscles or skeletal structure; most plants are able to reposition their leaves in order to receive more sunlight (a phenomenon referred to as "phototropism") - in the case of sunflowers, this occurs throughout the day in a phenomenon known as heliotropism $[4,5]$. The utility of elephant trunk-like technology is relatively clear: their design is inherently soft and can serve a wide range of purposes. However, the applicability of plant structures to soft robotics may be somewhat less clear. The primary attraction of many stimulus-responsive plants lies in how they are able to react (within variable timeframes) to external stimulus without muscles or skeletons; instead, these plants utilize an organization that makes little to no distinction between actuators (things that cause movement, e.g. muscles in animals) and structural elements (things that are moved by actuators - analogous to skeletons in vertebrates) [6]. This integration of structure and actuation is ideal for soft robotic systems, which rely on having a continuous range of motion throughout their members. As such, materials and mechanisms inspired by plant systems that are able to simultaneously sense and respond to external stimuli are the subject of much research, as they hold a great deal of potential for applications in biomedical, chemical, and mechanical engineering [7].

That being said, the animal kingdom still provides myriad sources of inspiration for robotic design, as previously mentioned. As pointed out by Kim et al., animals are largely composed of soft tissues, including muscle and other supportive tissues (e.g. digestive, respiratory, nervous, etc.), which allows them to perform complex movement patterns and adapt to different environments and situations [8]. Examples range from octopi to caterpillars to elephants, with many techniques and devices being inspired by each of these animals. As described previously, the kind of adaptability seen in these animals is key to the function of soft robots and allows them to be much more robust in their applications than traditional robots. Soft regions of the body allow for animals to dissipate impact force, mold to contact surfaces, and navigate gaps 
that would otherwise be too small [8]. All of these abilities stand to greatly benefit various areas of medicine and have numerous potential applications, from surgery to drug delivery $[2,9]$.

\subsection{Application Areas}

One aspect of medicine which stands to benefit from the use of soft robots is that of surgery, specifically minimally invasive surgery. Minimally invasive surgery (MIS) relies largely on the use of endoscopic instruments to complete procedures without creating large incisions [10]. MIS is seeing growing popularity, as it tends to reduce recovery time, patient discomfort, and the risk of future complications [11]. However, one of the most prominent issues with MIS stems from the endoscope itself - more specifically, the difficulty of navigating using these endoscopes [10]. Issues can arise due to the lack of vision inherent in these types of procedures, and as such, it is critical that surgeons have full and easy control of their instruments. Given the obstacles that must be navigated during these types of procedures, soft robots are clearly a desirable alternative to some of the stiffer instruments in use now [10].

Furthermore, certain devices show promise for applications in drug delivery, especially those which can be remotely activated. Such devices, such as the heat-responsive drug capsule designed by Bergueiro and Calderón, are less whole robots as actuators, responding to external stimuli to perform a function [12]. Nonetheless, such developments - especially those which integrate stimulus response with actuation - may be instrumental in the field of soft robotics by allowing such robots to act on their own.

\section{Recent Advances}

\subsection{Actuator Material Metrics}

Soft actuators can generally be differentiated based on two characteristics: the material of which they are composed and their mode of activation. Common actuator materials include gels, polymers, fluids, paper, and carbon, and common activation methods include heat, light, electrical, chemical, pressure, and magnetic activation [13]. It is worth emphasizing that, although this report focuses primarily on artificial soft actuators, there are other methods of soft actuation, including systems based on biomolecules, 
hybridized muscle cells, and microorganisms, as pointed out by Hines et al. [13]. Nonetheless, the field of artificial soft actuation is broad and continually growing, warranting an overview of the various actuation modes and materials.

As with all materials engineering applications, there are many aspects to consider when designing a material for a specific purpose and many metrics by which to judge them. Two common metrics are stress and strain; particular to soft actuator materials, these are measured as the stress and strain generated by the material during actuation. One common benchmark for material performance in terms of generating stress and strain is that of human skeletal muscle, which is capable of producing about $20 \%$ strain (although the maximum can exceed 40\%) and $100 \mathrm{kPa}$ of stress (maximum can exceed $350 \mathrm{kPa}$ ) [13]. Additionally, one must consider the material's actuation method, as this can place constraints on the actuator's size and performance [13]. Although sometimes difficult to generalize, this metric can be used to compare different materials to each other by examining how each one's force output scales with its length [13]. Finally, researchers engineering soft actuators must be mindful of the material's mode of activation, which may be limited based on the setting. Each mode of activation comes with its own set of benefits and detractions, however. For example, electrical, magnetic, and photonic activation can all be applied remotely (i.e. without any sort of tethering) from the site of the actual material and are easily modulated, whereas chemical activation is highly dependent upon the surrounding environment, which may or may not be desirable, depending on the application [13]. See Table 1 for a summary of the costs and benefits of each of the common modes of actuator activation.

Table 1: Comparing advantages and disadvantages of different modes of activation

\begin{tabular}{|l|l|l|}
\hline Mode of Activation & Benefits & Drawbacks \\
\hline Light [13] & $\begin{array}{l}\text { Remote application, easy modulation, } \\
\text { can be applied to highly specific areas }\end{array}$ & $\begin{array}{l}\text { May require exposure to high-energy } \\
\text { radiation }\end{array}$ \\
\hline
\end{tabular}




\begin{tabular}{|l|l|l|}
\hline Electric field [13] & $\begin{array}{l}\text { Remote application, easy modulation, } \\
\text { small setups with readily available }\end{array}$ & Delayed action (hysteresis) \\
& components & \\
\hline Magnetic field [13] & Remote application, easy modulation, & Setups tend to be large and expensive, \\
& penetrates most materials, greater & may interfere with other \\
& range of motion & instrumentation \\
\hline Heat [13] & Safer than UV or chemical (i.e. & Decreased speed and efficiency \\
\hline Fluid pressure [13] & Comparatively high force output for & Tethered to external pump or \\
& less weight & compressor \\
\hline Chemical change & No external power source, automatic & Must be operable in fluid or kept in a \\
& sensitivity to surrounding conditions & carefully controlled environment, slow \\
& & activation (often based on diffusion) \\
\hline 14$]$ &
\end{tabular}

\subsection{Gels and Polymers}

Gel and polymeric actuators are both capable of using a wide range of activation modes, and some are able to utilize multiple modes simultaneously [13]. Hydrogels as soft actuators are of particular interest due to their inherently high compliance and elasticity as well as their ionic conductivity and biocompatibility [15]. Hydrogels are composed of water and crosslinked polymers that form a highly elastic, optically transparent solid [15]. Since water is a large constituent of hydrogels, one can dissolve ions into hydrogels, rendering them highly conductive (Fig. 2) [15]. Furthermore, hydrogels can be engineered to be thermally

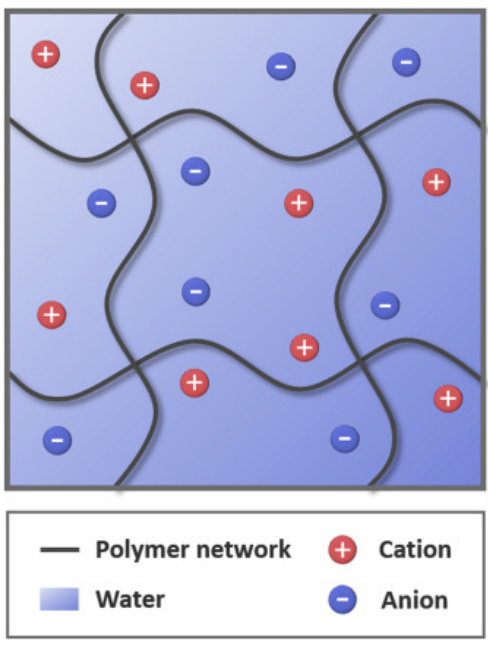

Figure 2: Hydrogels can contain ions dissolved in water, making them electrically conductive. Borrowed from Lee et al. [15].

responsive, as shown by the borax cross-linked polyvinyl alcohol 
derivative hydrogel produced by Gao et al. [16]. Such hydrogels have either lower critical solution temperature (LCST), in which case they shrink at temperatures above the critical temperature, or upper critical solution temperature (UCST), in which case they swell when above their critical temperature [15]. The bilayer hydrogel produced by Gao et al. takes on different shapes depending on temperature as well as the humidity of its environment [16]. As with most bilayer actuators, the two layers of this hydrogel are the active layer (i.e. the layer that actually expands/contracts in response to stimuli) and the passive layer (i.e. the layer that deforms in response to forces applied by the contraction/expansion of the active layer) [16]. The active layer of this particular hydrogel expands under conditions of low temperatures, and its expansion is amplified by higher humidity (see Fig. 3b-c) [16]. Likewise, it contracts at high temperatures, an effect which is exacerbated by lower humidity (see Fig. 3d-e) [16]. On a similar topic, Miriyev et al. showed that thermal actuation in hydrogels could be made more effective by implementing phase-change mechanics [17]. This mechanic involves integrating liquid-vapor bubbles (since liquids exist in a gaseous-liquid equilibrium with their environment) into the hydrogel and causing the bubbles to deform with changes in

(a)

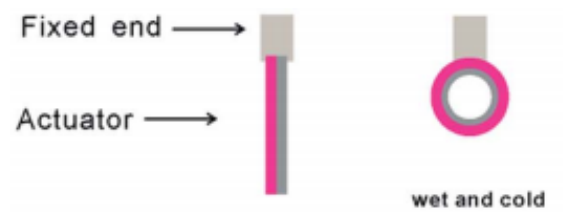

(c)

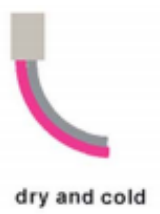

(d) $\uparrow$

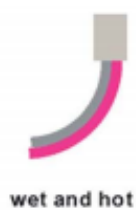

(e)

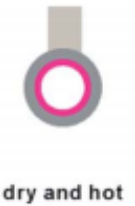

Figure 3: Structure and function of bilayer hydrogel produced by Gao et al. (a) The pink layer represents the active layer of the hydrogel; the gray layer represents the passive layer. (b-c) Cold temperatures cause the active layer to expand, an effect which is amplified by high humidity, causing the hydrogel to reach all the way up to the point of fixation. (d-e) Similarly, warm conditions cause the active layer to contract, an effect which is amplified by low humidity. Adapted from Gao et al. [16].

$\dagger$ Note that the layers in Fig. $4 d$ may be reversed; I believe the active layer should still be on the left side and the gray layer on the right. be on the left side and the gray layer on the right.

$$
\text { . }
$$

By using this mechanic, Miriyev

et al. were able to produce a hydrogel that produced rapid actuation while still providing a high degree of strain - two qualities which can be difficult to combine into the same material [17].

Chemically activated hydrogel actuators generally fall into one of three categories: solventresponsive, pH-responsive, or biomolecule-responsive [15]. Hydrogels that are photoactive generally 
utilize one of three underlying mechanisms: photoswitching, photothermal conversion, and light-activated hydrogel-cell hybrids. Photoswitching relies on photoswitchable moieties, molecules that are reversibly altered by exposure to light of a certain wavelength; on the other hand, photothermal conversion combines light activation with thermal activation to convert light energy to heat, most often via carbon nanotubes or gold nanoparticles $[15,18]$. The concept of cell hybridization is somewhat outside the scope of this paper, but in short, it combines living cells with the hydrogel to produce selective activation [15]. For example, Raman et al. integrated skeletal muscle cells with blue light-activated ion channels into a hydrogel, which caused the gel to contract when subjected to light of a certain wavelength [19]. Electrically responsive hydrogel actuators rely on either Maxwell stress - in which case they are called dielectric elastomer actuators (DEA) or electrically induced osmotic pressure [15]. Magnetic actuation is also possible for hydrogel actuators by dispersing magnetic particles in the hydrogel, which push on the hydrogel's crosslinker matrix when a magnetic field is applied, as demonstrated by Zhao et al. [20]. As will be discussed later, such actuators are promising for applications in drug delivery [15]. Hydraulically activated hydrogel actuators - i.e. actuators that swell and shrink in response to the pressure of the surrounding fluidic environment - have also been made, such as the hydrogel actuator produced by Yuk et al., which is able to achieve high actuation force in comparatively little time [21]. Finally, some hydrogels are also capable of actuation by several modes, as previously mentioned, such as that designed by Dong et al., which responds to both thermal and chemical stimuli [22].

Other polymeric actuators can be classified as either liquid-crystal (LC) materials or shape-memory polymers (SMPs) [13]. Liquid crystal molecules are referred to as mesogens and are characterized as being polar, stiff, rod-shaped molecules that readily reorient when placed in an electric field [13]. When these mesogens polymerize, they can form liquid-crystal polymers (LCPs), liquid-crystal polymer networks (LCNs), or liquid-crystal elastomers (LCEs) [13]. LCNs and LCEs generally receive more attention than LCPs in terms of actuator materials and are differentiated by their mechanical properties [13]. LCNs display moderate to high degrees of crosslinking, making them less elastic but also more capable of mesogen 
alignment [13]. LCEs are characterized by a lower degree of crosslinking than LCNs and as a result show greater elasticity (similar to rubber) but also offer less ability to align their mesogens [13]. Another aspect of LC material that affects their applications - in particular, which modes of actuation are possible for them - is the material's phase. Two of the most important LC phases for actuation purposes are nematic or smectic [13]. In nematic LCs, the mesogens are aligned in the same direction, but they aren't necessarily arranged in rows [13]. Smectic LCs, on the other hand, have their mesogens aligned in the same general direction, and the mesogens are arranged in rows [13]. When heated, nematic-phase LCs are capable of reversible contraction caused by a transition of the material's mesogens to an unordered, randomly oriented state; when in this state, the material is said to be in an isotropic phase [13]. As demonstrated by Lehmann et al., actuation is also possible with smectic LCs by placing ferroelectric LCEs in an electric field [23].

Finally, certain polymers, referred to as shape-memory polymers, are able to "remember" and return to a shape despite deformation, although polymers can be kept in a deformed state by crystallizing or vitrifying the polymer [13]. "Freezing" the polymer in this manner is reversible (e.g. by heat application) and takes advantage of the polymer's glass transition temperature $\left(T_{g}\right)$; this property of shape-memory polymers allows them to temporarily store strain for release later via thermomechanical cycling. This process operates by deforming the polymer while it is above its $T_{g}$, holding it in that position while its temperature is lowered below its $T_{g}$, leaving it in that position for as long as desired (at this point the deformation, or strain, is stored for future use), and finally raising the polymer above its $T_{g}$, causing it to release the stored strain and assume its original, "memorized" shape [13]. In very select cases storing strain in a polymer does not require external force, but for most polymers, manual deformation or constant loading are necessary to store strain [13].

\subsection{Fluids, Paper, and Carbon}

Fluid-based actuators generally rely on pressure changes, viscosity changes, surface tension-based activation at the fluid boundary, or a combination thereof [13]. Although these mechanisms generally come with several advantages, such as high-speed changes and high effectiveness on small scales, they also come 
with several drawbacks, including being unable to be repeatedly used due to leakage, accidental mishandling of the object being moved by the fluid droplet, potential unintended mixing of fluids, and high dependence on and sensitivity to environmental conditions [13]. However, it is possible to address many of these issues via encapsulation of the liquids being used or the use of liquid marbles, a phenomenon developed by Aussillous et al. in which a droplet is coated with hydrophobic particles, keeping it from interacting with the surface [24]. One of the most intriguing features of these liquid marbles is that, when designed properly, they have the capacity to behave in a very similar manner to living cells; dividing, supporting low-level metabolism, evolving, and communicating with one another; these specialized liquid marbles are referred to as active droplets [25].

A rather recent, albeit unassuming, entrant into the field of soft actuators is one of the most prevalent commodities in academia: paper. One application is the development of a diagnostic "paper machine" by Connelly et al., which uses layered paper microfluidics to detect the presence of $E$. coli in a blood sample (see Fig. 4) [26]. The advantages of using paper as a building material are readily apparent: in addition to being biodegradable and lightweight, paper is cheap and easily obtainable, making it an ideal material for making low-cost devices for low-income regions, as mentioned by Connelly et al. [26]. In conjunction with its newfound role as a possible soft actuator material, paper's ability to conduct many liquids via capillary action stands to bring it to the forefront of low-cost medical manufacturing.

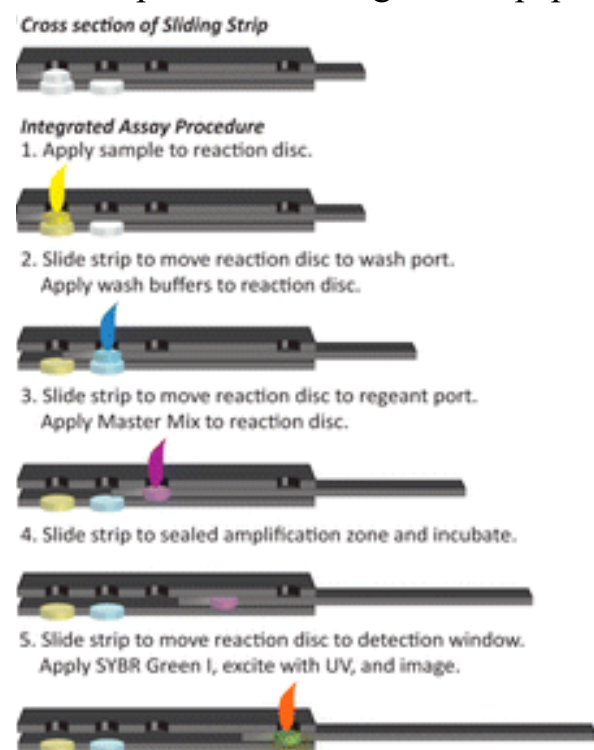

Figure 4: Diagram of how the "paper machine" works. Adapted from Connelly et al. [26].

The final actuator material to be covered here - carbon - may not initially seem to belong in a discussion about soft robotics. After all, carbon is generally known for its stiffness and strength, such as when it is used in applications such as carbon fiber-reinforced polymer, which is designed specifically to be as noncompliant as possible - the opposite of what soft-robot designers look for in a material. However, 
carbon particles, graphene, and carbon nanotubes (CNTs) have all found a place in the field of soft actuators, largely on account of their ability to conduct heat and electricity [13]. CNTs are most commonly used, and they exist in two forms: single-walled and multi-walled CNTs [13]. Both multi-walled CNTs, which are essentially nested groups of single-walled CNTs, and single-walled CNTs are conductive along their lengths but are highly insulative perpendicular to their length, allowing for many design possibilities [13]. CNTs are not very conducive to strain, but they can be folded into sheets or yarns, allowing them to function as soft actuators, as shown by Baughman et al. [27]. The work of Baughman et al. produced single-walled, electrically activated CNT sheets capable of greater force output than human skeletal muscle (0.75 MPa vs. $0.3 \mathrm{MPa}$ ) [27]. However, nanotubes can be somewhat expensive to purchase, with prices for single-walled CNTs ranging from 100-300 USD per gram [28, 29].

Table 2: Summary of different materials' actuating capabilities.

\begin{tabular}{|l|l|l|l|l|l|l|}
\hline Material & Light & Electric & Magnetic & Heat & Fluid & Chemical \\
& & Field & Field & & Pressure & Change \\
\hline Polymers & Yes & Yes & Yes & Yes & Yes & Yes \\
\hline Gels & Yes & Yes & Yes & Yes & Yes & Yes \\
\hline Fluid & Yes & Yes & Yes & Yes & Yes & Yes \\
\hline Paper Structures & No & Yes & Yes & Yes & No & Yes \\
\hline Carbon & No & Yes & No & Yes & Yes & No \\
\hline
\end{tabular}

\subsection{Biocompatible Soft Actuators}

A critical aspect of actuators designed for use in biomedical applications - especially those which will come into direct contact with living tissue - is that they must be biocompatible. Although cellulose has been shown to be a viable option for creating electroactive polymers (EAPs), its biomedical applications in this particular sense are at present rather limited, as it also requires the use of heavy metals as electrodes, 
making them highly incompatible with biomedical applications [30]. However, Lu and Chen have designed an EAP system that avoids the use of toxic heavy metals and instead relies upon chitosan, carbon nanotubes, and ionic liquids, all of which are biocompatible [30]. The benefit of using chitosan is that it is a naturally occurring, cationic polymer, so it is common to see carbon nanotubes (CNTs) functionalized with chitosan when designed for actuation purposes [30]. Furthermore, these chitosan-CNT complexes demonstrate beneficial electrochemical and electromechanical properties when combined with ionic liquids, producing a gel substance which can be evaporated to form a film to separate the two electrodes of the polymer [30, 31]. Lu and Chen also found that the electrode membranes composed of the chitosan-CNT complex adhered well to the ionic liquid electrolytic membrane, causing them to stay attached without assistance [30]. In practice, opposite charges are applied to each electrode, and the full composite bends one way or the other, depending on how the electrode layers are charged [30]. The whole composite bends towards the positively charged terminal due to steric interference from mobile ions [30]. This occurs as a result of 1-butyl-3methylimidazolium $\left(\mathrm{BMI}^{+}\right)$cations travelling to the negatively charged cathode while the smaller tetrafluoroborate $\left(\mathrm{BF}_{4}^{-}\right)$anions travel to the positively charged anode; this ion displacement results in steric interference between the larger $\mathrm{BMI}^{+}$cations and causing them to push against one another and expand the cathodic electrode [30].

Gao et al. were also able to manufacture a bilayer hydrogel actuator based on biocompatible components, such as polyvinyl alcohol (PVA) [16]. As previously discussed, this actuator utilizes a bilayer structure, wherein one layer is active (i.e. capable of deformation in response to activation) and the other layer is passive (i.e. only capable of deformation by forces exerted by the active layer) [16]. This actuator is also thermally reactive, with the active layer expanding in response to lower temperatures (accentuated by high humidity) and shrinking in response to higher temperatures (accentuated by low humidity) [16]. The primary cause for humidity playing the role it does is that a dry environment dehydrates the hydrogel, causing it to shrink, whereas a humid environment causes the hydrogel to retain or even take up water, resulting in swelling [16]. One potential issue with this effect is that the humidity of one's work environment 
cannot always be controlled, so having an actuator that is considerably sensitive to humidity could be problematic. This is especially true for in vivo biological applications, as living tissue and organs are, of course, very damp. Regardless, the hydrogel provides considerable resistance to fatigue, as it is able to hold a deformed shape for more than two hours without weakening [16]. Additionally, this material is able to carry several times its own weight, as the authors showed by using a two-milligram hydrogel actuator to lift a 23-milligram mass two centimeters in less than two minutes [16]. The high loading capacity (i.e. ratio of mass lifted to mass of device) and fast actuation time demonstrated by this material makes it a potential solution for artificial muscle, outperforming many similar actuators in terms of speed and/or capacity, such as the one created by Islam et al. $[16,32]$. The team of Islam et al. produced a similar multilayer actuator to that designed by Gao et al., using a gold-coated substrate as a passive layer and covering it with a gel layer that expands and contracts in response to humidity, similar to the actuation mechanism used by the hydrogel actuator designed by Gao et al. [32]. As both of these actuators are highly humidity-dependent, their in-vivo applications are limited, as previously discussed. However, it is still possible for these actuators to serve a purpose outside of the body and in a more easily controlled environment.

\subsection{Remotely Activated Actuators}

Very recently, the team of Novelino et al. designed an origami-based structure that could deform based on the application of an external magnetic field [9]. The significance of their design lies primarily in its mode of actuation. Although origami has seen application in actuator engineering for years, it often requires a bulky mechanism to actuate, such as pneumatic or mechanical actuation, making unconducive to small-scale use in vivo [9]. However, by creating an actuator that is capable of deforming under an externally applied magnetic field, Novelino et al. have circumvented this issue. This design utilizes the Kresling origami pattern developed by Kresling et al., which allows for a cylindrical shape to expand or collapse $[33,34]$. The use of this pattern in the device allows it to switch between two stable geometric states extremely rapidly - changing

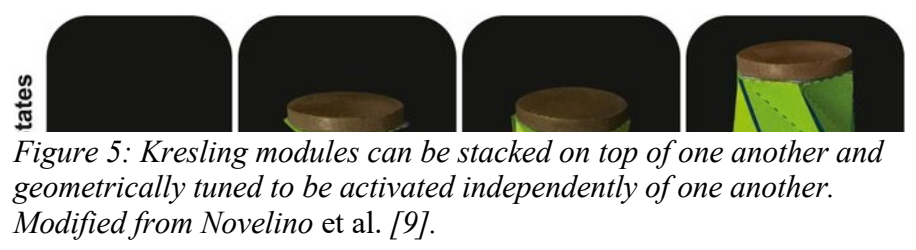


forms in tenths of a second $[9,35]$. This device's small size, rapid activation time, remote mode of activation, and ability to be modularly connected and activated (see Fig. 5) make it an attractive option for robotic drug delivery and non- or minimally invasive treatments, as described by co-author Renee Zhao in an interview by the Ohio State News (Ohio State University) [35]. According to Zhao, potential this technology has the potential to effectively navigate the body and, when triggered by an external magnetic field, deploy a drug payload at a particular site of the body [35]. The report's authors believe this device has additional applications in digital computing as basic logic circuits or computational memory [9].

Of course, origami actuators aren't the only materials to take advantage of magnetic activation. As mentioned earlier, Zhao et al. designed a ferromagnetic gel scaffold that mechanically responded to the application of an external magnetic field [20]. The applications of this scaffold lie primarily in the realm of drug distribution and controlled drug release, with the drug-laden gel acting like a sponge of water that can be squeezed out by the application of a magnetic field [20]. That said, it is worth noting that this scaffold is also compatible with cell seeding, a rarity among ferrogel materials [20]. The gel itself is composed of alginate and is modified with peptides containing RGD sequences (arginine-glycine-aspartate) to promote cell binding [20]. To give it its ferromagnetic properties, the gel is embedded with iron oxide nanoparticles approximately ten nanometers in diameter [20]. Finally, freezedrying is used to create pores of consistent size throughout the gel by evaporating water from the gel, leaving behind a system of interconnected macropores [20]. The most important part of this step is the size of the pores created. While many contemporary ferrogels have extremely small pore sizes (usually in the range of nanometers), the gels created by Zhao et al. contain pores on the micrometer scale, allowing larger molecules to be stored within them than most predecessor ferrogels [20]. Testing of the novel ferrogels also showed that they were capable of substantially greater

(a)

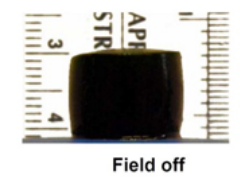

(b)
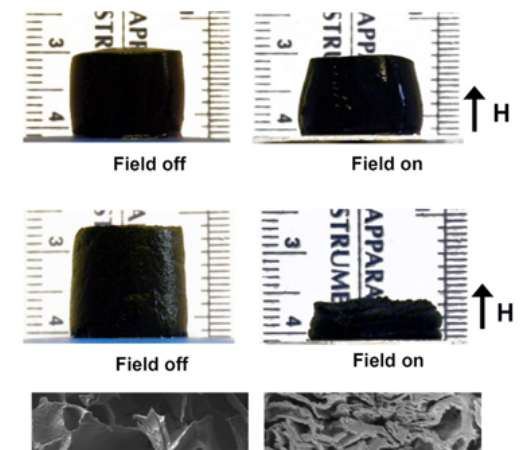

Figure 6: Deformation of ferrogels resulting from application of a magnetic field from below. (a) A standard example of a nanoporous ferrogel demonstrates $\sim 5 \%$ height decrease under the magnetic field. (b) The novel, macroporous ferrogel displays $\sim 70 \%$ height decrease under the magnetic field. (c) SEM images of the pores within the novel, macroporous ferrogel with and without the application of a magnetic field $($ scale bar $=500 \mu \mathrm{m})$. Modified from Zhao et al. [20]. 
deformation as compared to previous ferrogel formulations, with the novel gel displaying a $70 \%$ reduction in size compared to a $5 \%$ reduction seen in a "typical nanoporous ferrogel" (see Fig. 6) [20, 36]. Zhao et al. also found that repeated flushing of the ferrogels via cycling magnetic fields greatly improved drug release (see Fig. 7), likely due to the shear forces provided by liquid being forced through the gels' pores as they were periodically flushed [20]. One molecule tested for release improvement via flushing was mitoxantrone, which forms an ionic complex with the alginate

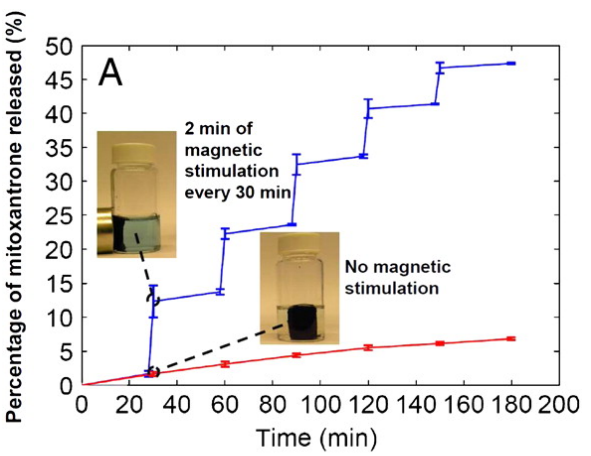

Figure 7: Flushing the ferrogel via a cycling magnetic field greatly improves release of mitoxantrone, indicated by the PBS solution turning blue. The flushing process consists of subjecting a ferrogel to two minutes of 120 on/off magnetic field cycles, with this twominute process occurring every 30 minutes. Borrowed from Zhao et al. [20]. making it particularly slow to release; other test molecules included plasmid DNA and SDF-1 $\alpha$ (a chemokine) [20]. All molecules tested displayed a dramatic increase in molecule release, although the amount released and the time between cycles varied for each molecule [20]. Similar results were seen in in-vivo tests of the gel using mouse models implanted with a stain-loaded ferrogel, with the amount of fluorescent stain released being much greater for the magnetically stimulated ferrogels compared to the control (unstimulated) animals [20]. Sapir et al. also found that magnetic cycling was beneficial for seeding cardiac muscle cells, as the repeated mechanical stimulation improved cell organization and overall cardiac tissue formation [37]. Using a very similar formulation to the one described by Zhao et al. (alginate infused with magnetic nanoparticles and coated with RGD peptides), Sapir et al. successfully manufactured a cardiac patch by seeding the ferrogel scaffold with neonatal rat cardiac cells and then incubating these scaffolds within an alternating magnetic field, showing that the ferrogel designed by Zhao et al. has even greater biomedical potential than initially supposed, with additional applications tissue engineering [37].

So far, all remotely activated actuators described in this section have relied upon magnetization, but there are certainly other viable methods of activation in use, as shown by Yang et al., who designed a soft actuator activated by infrared light [38]. The actuator uses a bilayer structure, with one infrared-active layer and another transparent, passive layer; importantly, these two layers were designed to have 
(a)

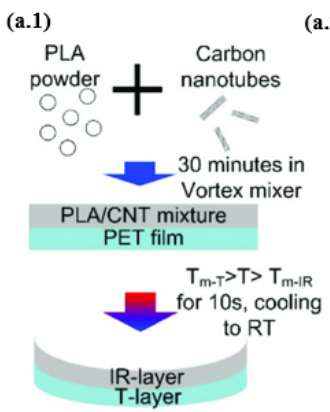

(b)

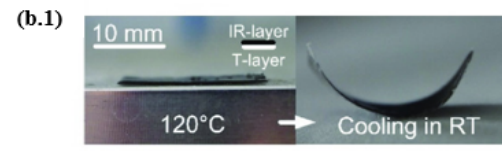

(b.2)

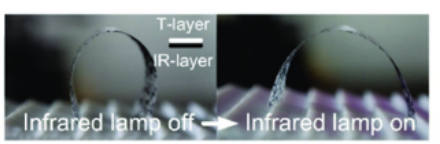

(b.3)

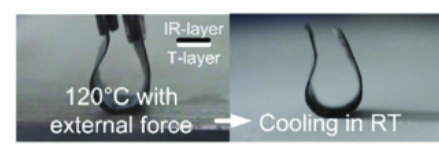

(a.3)

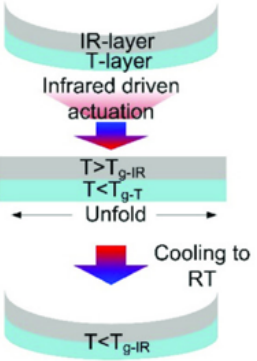

(c)

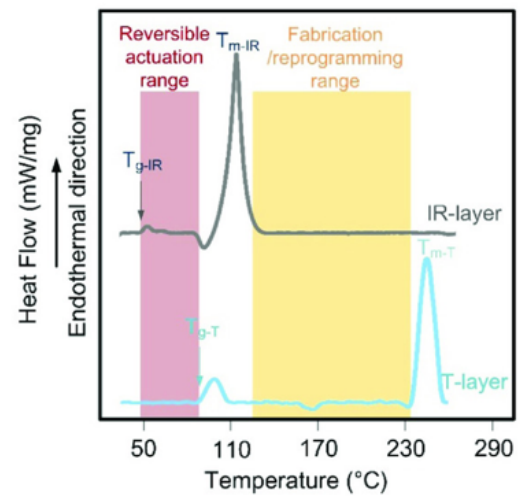

Figure 8: (a.1) Actuator manufacturing process. The infrared-active layer ("IRlayer") is composed of polylactic acid (PLA) and carbon nanotubes (CNTS), and the transparent layer ("T-layer") is composed of polyethylene terephthalate (PET). (a.2) Actuation via infrared irradiation, followed by relaxation via cooling. (a.3) Diagram of device "reprogramming". (b.1) Fabrication of actuator. (b.2) Actuation of device via infrared irradiation. (b.3) "Reprogramming" of device. (c) differential scanning calorimetry curves for both layers of device. Figure modified from Yang et al. [38].

significantly different glasstransition and melting temperatures between each other [38]. The difference between the layers' glass transition temperatures allows for the device to be reversibly actuated, as the infrared source induces deformation by heating the infraredactive layer [38]. On the other hand, the difference in melting points allows the device to be "reprogrammed," that is, reshaped (see Fig. 8) while still retaining its actuation capabilities [38].

Designing this device to use infrared light for actuation - rather than ultraviolet light or visisble lasers - provides several benefits, such as greater safety and penetration depth, in addition to making it cost-effective, as infrared lamps are relatively inexpensive [38]. Furthermore, this actuator is composed of known biocompatible materials - polylactic acid (PLA), carbon nanotubes, and polyethylene terephthalate (PET) - meaning that it is likely to be a viable solution for in-vivo soft actuator applications [38, 39]. Although the applications of this technology are currently unclear, this type of device may be well-suited for minimally-invasive surgeries, as discussed earlier, due to its reprogramability and mode of actuation.

\section{Conclusion}

Soft robots are one of the most promising up-and-coming technologies in medicine, potentially solving many problems, from oversized robotic surgery apparatuses to a lack of flexible instruments for 
minimally-invasive surgery. However, an important aspect of the pursuit of these devices is the material used to construct them. While traditional, hard robots utilize discrete actuators to move their members, soft robots blur the line between structural members and actuators, as the two are often one in the same. As such, the materials composing soft robots - specifically the mobile portions - are referred to as soft actuators, due to their inherently soft and compliant nature. As with hard-robotic actuators, there are many different modes of activation for soft actuators, including light, chemical, magnetic, and thermal activation. In addition to being flexible and capable of navigating complex geometry (such as the interior of the body), soft actuators can be remotely actuated in some cases, making them of great potential value for drug delivery, minimally invasive surgery, endoscopies, and other in-vivo medical applications.

Throughout this paper, various soft-actuator materials have been discussed, with an emphasis on application to medicine. Actuator materials can consist of several different materials, such as hydrogels, polymers, fluids, and even paper. Likewise, they can be divided up based on their mode of actuation, with some requiring direct contact with a stimulus or outside apparatus (as with most electrically active soft actuators) and many others capable of actuation using a remotely applied stimulus (as with magnetic actuation). Each material and mode of actuation has its own set of benefits and drawbacks, but as research in this field continues, there will no doubt be even greater advances made, with maximized benefit and minimized limitations. Most exciting will be seeing how these advances are applied to further the future of medicine. 


\section{References}

[1] N. G. Hockstein, C. G. Gourin, R. A. Faust and D. J. Terris, "A history of robots: from science fiction to surgical robotics," Journal of Robotic Surgery, vol. 1, no. 2, pp. 113-118, 2007.

[2] D. Trivedi, C. D. Rahn, W. M. Kier and I. D. Walker, "Soft robotics: Biological inspiration, state of the art, and future research," Applied Bionics and Biomechanics, 2008.

[3] B. O'Brien, "Master The Fundamentals Of Stretch-Sensor Sensitivity," 11 Nov 2016. [Online]. Available: https://www.fierceelectronics.com/components/master-fundamentals-stretch-sensorsensitivity. [Accessed 16 Oct 2020].

[4] R. Pomeroy, "7 Facts You Didn't Know About Elephant Trunks," 14 Oct 2013. [Online]. Available: https://www.realclearscience.com/blog/2013/10/the-most-amazing-appendage-in-the-world.html. [Accessed 17 Oct 2020].

[5] H. S. Atamian, N. M. Creux, E. A. Brown, A. G. Garner, B. K. Blackman and S. L. Harmer, "Circadian regulation of sunflower heliotropism, floral orientation, and pollinator visits," Science, vol. 353, no. 6299, pp. 587-590, 2016.

[6] S. Li and K. W. Wang, "Plant-inspired adaptive structures and materials for morphing and actuation: a review," Bioinspiration \& Biomimetics, vol. 12, no. 1, 2016.

[7] Q. Guo, E. Dai, X. Han, S. Xie, E. Chao and Z. Chen, "Fast nastic motion of plants and bioinspired structures," Journal of The Royal Society Interface, vol. 12, no. 110, 2015.

[8] S. Kim, C. Laschi and B. Trimmer, "Soft robotics: a bioinspired evolution in robotics," Trends in Biotechnology, vol. 31, no. 5, pp. 287-294, 2013.

[9] L. S. Novelino, Q. Ze, S. Wu, G. H. Paulino and R. Zhao, "Untethered control of functional origami microrobots with distributed actuation," Proceedings of the National Academy of Sciences of the United States of America, vol. 117, no. 39, pp. 24096-24101, 2020.

[10] M. Runciman, A. Darzi and G. P. Mylonas, "Soft Robotics in Minimally Invasive Surgery," Soft Robotics, vol. 6, no. 4, pp. 423-443, 2019.

[11] Mayo Clinic Staff, "Minimally invasive surgery," Mayo Clinic, 24 Aug. 2019. [Online]. Available: https:/www.mayoclinic.org/tests-procedures/minimally-invasive-surgery/about/pac-20384771. [Accessed 19 Oct. 2020].

[12] J. Bergueiro and M. Calderón, "Thermoresponsive Nanodevices in Biomedical Applications," Macromolecular Bioscience, vol. 15, no. 2, pp. 183-199, 2014.

[13] L. Hines, K. Petersen, G. Z. Lum and M. Sitti, "Soft Actuators for Small-Scale Robotics," Advanced Materials, vol. 29, no. 13, 2017. 
[14] G. Wu, X. Wu, Y. Xu, H. Cheng, J. Meng, Q. Yu, X. Shi, K. Zhang, W. Chen and S. Chen, "HighPerformance Hierarchical Black-Phosphorous-Based Soft Electrochemical Actuators in Bioinspired Applications," Advanced Materials, vol. 31, no. 25, 2019.

[15] Y. Lee, W. J. Song and J.-Y. Sun, "Hydrogel soft robotics," Materials Today Physics, vol. 15, p. $100258,2020$.

[16] L. Gao, G. Guo, M. Liu, Z. Tang, L. Xie and Y. Huo, "Multi-responsive, bidirectional, and large deformation bending actuators based on borax cross-linked polyvinyl alcohol derivative hydrogel," RSC Advances, vol. 7, no. 63, pp. 40005-40014, 2017.

[17] A. Miriyev, K. Stack and H. Lipson, "Soft material for soft actuators," Nature Communications, vol. 8, no. 596, 2017.

[18] X. Kang, X. Guo, X. Niu, W. An, S. Li, Z. Liu, Y. Yang, N. Wang, Q. Jiang, C. Yan, H. Wang and Q. Zhang, "Photothermal therapeutic application of gold nanorods-porphyrin-trastuzumab complexes in HER2-positive breast cancer," Scientific Reports, vol. 7, p. 42069?, 2017.

[19] R. Raman, C. Cvetkovic, S. G. M. Uzel, R. J. Platt, P. Sengupta, R. D. Kamm and R. Bashir, "Optogenetic skeletal muscle-powered adaptive biological machines," Proceedings of the National Academy of Sciences of the United States of America, vol. 113, no. 13, pp. 3497-3502, 2016.

[20] X. Zhao, J. Kim, C. A. Cezar, N. Huebsch, K. Lee, K. Bouhadir and D. J. Mooney, "Active scaffolds for on-demand drug and cell delivery," Proceedings of the National Academy of Sciences of the United States of America, vol. 108, no. 1, pp. 67-72, 2011.

[21] H. Yuk, S. Lin, C. Ma, M. Takaffoli, N. X. Fang and X. Zhao, "Hydraulic hydrogel actuators and robots optically and sonically camouflaged in water," Nature Communications, vol. 8, p. 14230, 2017.

[22] L. Dong, A. K. Agarwal, D. J. Beebe and H. Jiang, "Adaptive liquid microlenses activated by stimuliresponsive hydrogels," Nature, vol. 442, no. 7102, pp. 551-554, 2006.

[23] W. Lehmann, H. Skupin, C. Tolksdorf, E. Gebhard, R. Zentel, P. Krüger, M. Lösche and F. Kremer, "Giant lateral electrostriction in ferroelectric liquid-crystalline elastomers," Nature, vol. 410, no. 6827, pp. 447-450, 2001.

[24] P. Aussillous and D. Quéré, "Properties of liquid marbles," Proceedings of the Royal Society A: Mathematical, Physical and Engineering Sciences, vol. 462, no. 2067, 2006.

[25] S. Lach, S. M. Yoon and B. A. Grzybowski, "Tactic, reactive, and functional droplets outside of equilibrium," Chemical Society Reviews, vol. 45, pp. 4766-4796, 2016.

[26] J. T. Connelly, J. P. Rolland and G. M. Whitesides, "'Paper Machine" for Molecular Diagnostics," Analytical Chemistry, vol. 87, no. 15, p. 7595-7601, 2015.

[27] R. H. Baughman, C. Cui, A. A. Zakhidov, Z. Iqbal, J. N. Barisci, G. M. Spinks, G. G. Wallace, A. Mazzoldi, D. D. Rossi, A. G. Rinzler, O. Jaschinski, S. Roth and M. Kertesz, "Carbon Nanotube Actuators," Science, vol. 284, no. 5418, pp. 1340-1344, 1999. 
[28] Cheap Tubes, "Single Walled Carbon Nanotubes Products," Cheap Tubes, 2020. [Online]. Available: https://www.cheaptubes.com/product-category/single-walled-carbon-nanotubes/. [Accessed 1711 2020].

[29] Ossila, "Single-Walled Carbon Nanotubes, SWCNT," Ossila, 2020. [Online]. Available: https://www.ossila.com/products/single-walled-carbon-nanotubes?variant=34447448961. [Accessed $17112020]$.

[30] L. Lu and W. Chen, "Biocompatible Composite Actuator: A Supramolecular Structure Consisting of the Biopolymer Chitosan, Carbon Nanotubes, and an Ionic Liquid," Advanced Materials, vol. 22, no. 33, pp. 3745-3748, 2010.

[31] T. Fukushima and T. Aida, "Ionic Liquids for Soft Functional Materials with Carbon Nanotubes," Chemistry - A European Journal, vol. 13, no. 18, pp. 5048-5058, 2007.

[32] M. R. Islam, X. Li, K. Smyth and M. J. Serpe, "Polymer-Based Muscle Expansion and Contraction," Angewandte Chemie International Edition, vol. 52, no. 39, pp. 10330-10333, 2013.

[33] T. C. Hull and B. Kresling, "Folded tubes as compared to Kikko ('Tortoise-Shell') bamboo," Origami: Proceedings of the Third International Meeting of Origami Science, Mathematics, and Education, p. 197, 2002.

[34] Q. Zhang, J. Cai, M. Li and J. Feng, "Bistable behaviour of a deployable cylinder with Kresling pattern," 7th International Meeting on Origami in Science, Mathematics and Education (7OSME), 2018.

[35] L. Arenschield, "Soft robots, origami combine for potential way to deliver medical treatments," Ohio State News, 22 Sept 2020. [Online]. Available: https://engineering.osu.edu/news/2020/09/softrobots-origami-combine-potential-way-deliver-medical-treatments. [Accessed 10 Dec 2020].

[36] S.-H. Hu, T.-Y. Liu, D.-M. Liu and S.-Y. Chen, "Nano-ferrosponges for controlled drug release," Journal of Controlled Release, vol. 121, no. 3, pp. 181-189, 2007.

[37] Y. Sapir, B. Polyak and S. Cohen, "Cardiac tissue engineering in magnetically actuated scaffolds," Nanotechnology, vol. 25, no. 1, p. 4009, 2014.

[38] R. Yang, M. Jin, M. Jin, H. Qian, Q. Gao, G. Jin and S. Zhang, "Reprogrammable Untethered Actuator for Soft Bio-Inspired Robots," Advanced Intelligent Systems, p. 2000146 (Online Version of Record before inclusion in an issue), 2020.

[39] S. Racca, "What Are Biocompatible Polymers?," Drake Medical Plastics, 6 Aug 2020. [Online]. Available: https://www.drakemedicalplastics.com/2020/08/06/what-are-biocompatible-polymers/. [Accessed 11 Dec 2020].

[40] W. Mcmahan, M. A. Csencsits, V. K. Chitrakaran and D. Dawson, "Field trials and testing of the OctArm continuum manipulator," Proceedings - IEEE International Conference on Robotics and Automation, pp. 2336 - 2341, 2006. 
[41] Z. Zhang, L. Liu, J. Fan, K. Yu, Y. Liu, L. Shi and J. Leng, "New silicone dielectric elastomers with a high dielectric constant," Proceedings of SPIE - The International Society for Optical Engineering, vol. 6926, p. 692610, 2008.

[42] Y.-W. Kang, J. Woo, H.-R. Lee and J.-Y. Sun, "A mechanically enhanced electroactive hydrogel for 3D printing using a multileg long chain crosslinker," Smart Materials and Structures, vol. 28, no. 9, p. 5016, 2019.

[43] C. Jianguo, L. Yangqing, M. Ruijun, F. Jian and Z. Ya, "Nonrigidly Foldability Analysis of Kresling Cylindrical Origami," Journal of Mechanisms and Robotics, vol. 9, no. 4, pp. 41018-41028, 2017.

On my honor, I have neither given nor received any unauthorized aid in the completion of this exercise. 